\title{
MICROFTALMIA EN AOUILOEURYCEA CAFETALERA (CAUDATA: PLETHODONTIDAE) Y ECTROMELIA DE FÉMUR EN CRAUGASTOR RHODOPIS (ANURA: CRAUGASTORIDAE) OBSERVADAS EN UN BOSQUE DE NIEBLA DE VERACRUZ, MÉXICO
}

\author{
MICROPHTALMIA IN AQUILOEURYCEA CAFETALERA (CAUDATA: PLETHODONTIDAE) AND ECTROMELIA OF FEMUR \\ IN CRAUGASTOR RHODOPIS (ANURA: CRAUGASTORIDAE) OBSERVED IN A CLOUD FOREST IN VERACRUZ, MEXICO. \\ Juan Manuel Díaz-García ${ }^{1 *}$, Sarai Gómez-Toxqui², Enrique Silva-Ayala², Alfonso Kelly- \\ HERNÁNDEZ ${ }^{3}$ AND VÍCTOR VÁSQUEZ-CRUZ \\ ${ }^{1}$ Red de Biología y Conservación de Vertebrados, Instituto de Ecología, A.C. Carretera Antigua a Coatepec No. 351, Colonia El Haya, Xalapa, Veracruz, \\ México. C.P. 91000. \\ ${ }^{2}$ Facultad de Ciencias Biológicas, Benemérita Universidad Autónoma de Puebla. Avenida San Claudio Edificio 112-A Ciudad Universitaria, Colonia \\ Jardines de San Manuel, Puebla, Puebla. México. C.P. 72570 \\ ${ }^{3}$ PIMVS Herpetario Palancoatl. Avenida 19 No 5225, Colonia Nueva Esperanza, Córdoba, Veracruz, México. C.P. 94540. \\ "Correspondence: juanm.diazgarcia@gmail.com
}

Abstract.- In this note we present the first case reported of microphtalmia in Aquiloeurycea cafetalera and ectromelia of the femur in Craugastor rhodopis observed in a cloud forest of central Veracruz, Mexico.

Keywords.- amphibians, morphological abnormalities, deformations, malformations.

Resumen.- En esta nota presentamos el primer caso reportado de microftalmia en Aquiloeurycea cafetalera y de ectromelia de fémur en Craugastor rhodopis observadas en un bosque de niebla del centro de Veracruz, México.

Palabras clave.- anfibios, anormalidades morfológicas, deformaciones, malformaciones.

Una anormalidad morfológica es definida como cualquier desviación del intervalo normal de variación anatómica (Johnson et al., 2001), que puede ser causada por mutaciones, fallas en el desarrollo embrionario o traumatismos (SotoRojas et al., 2017). En anfibios se han reportado más de 20 diferentes tipos de anormalidades morfológicas (Meteyer, 2000; Peltzer et al., 2011), siendo las más comunes la falta de dígitos o extremidades (Blaustein \& Johnson, 2003). Los principales factores ambientales estresantes que están relacionados con la presencia de anormalidades en anfibios son los endoparásitos, los agroquímicos, la depredación selectiva, la endogamia y la radiación ultravioleta (Ankley et al., 2004; Johnson \& Lunde, 2005; Gray \& Lethaby, 2010).

En México son pocos los estudios que han registrado anormalidades morfológicas en anfibios, solo se han reportado casos observados en campo en las especies Ambystoma mexicanum (Robles-Mendoza et al., 2009), Ambystoma ordinarium (Soto-Rojas et al., 2017), Ambystoma velasci (Cruz-Pérez et al., 2009), Lithobates neovolcanicus (Barragán-Ramírez \& Navarrete-Heredia, 2011), Dryophytes arenicolor, Lithobates forreri, Lithobates zweifeli (MonroyVilchis et al., 2015) y Rhinella horribilis (Domínguez-Moreno et al., 2018). Asimismo, Aguillón-Gutiérrez y Ramírez-Bautista (2015), a partir de observaciones en campo y experimentos en laboratorio, reportaron que el contacto con hierro y plomo, dos metales pesados contaminantes, produce anormalidades en individuos de la especie Dryophytes plicatus. Estudiar las anormalidades en los anfibios puede ofrecer información acerca de la sensibilidad que tienen a la degradación de sus hábitats y de su importancia como indicadores de la calidad de los ecosistemas (Aguillón-Gutiérrez \& Ramírez-Bautista, 2015).

En este trabajo, presentamos el primer registro de microftalmia en la salamandra Aquiloeurycea cafetalera (ParraOlea et al., 2010) y de ectromelia de fémur en la rana Craugastor rhodopis (Cope, 1867), observados en un remanente de bosque de niebla de 30 ha perteneciente a la cooperativa Las Cañadas en el municipio de Huatusco, Veracruz, México (19.1930 N, $96.9954^{\circ} \mathrm{W}$ 


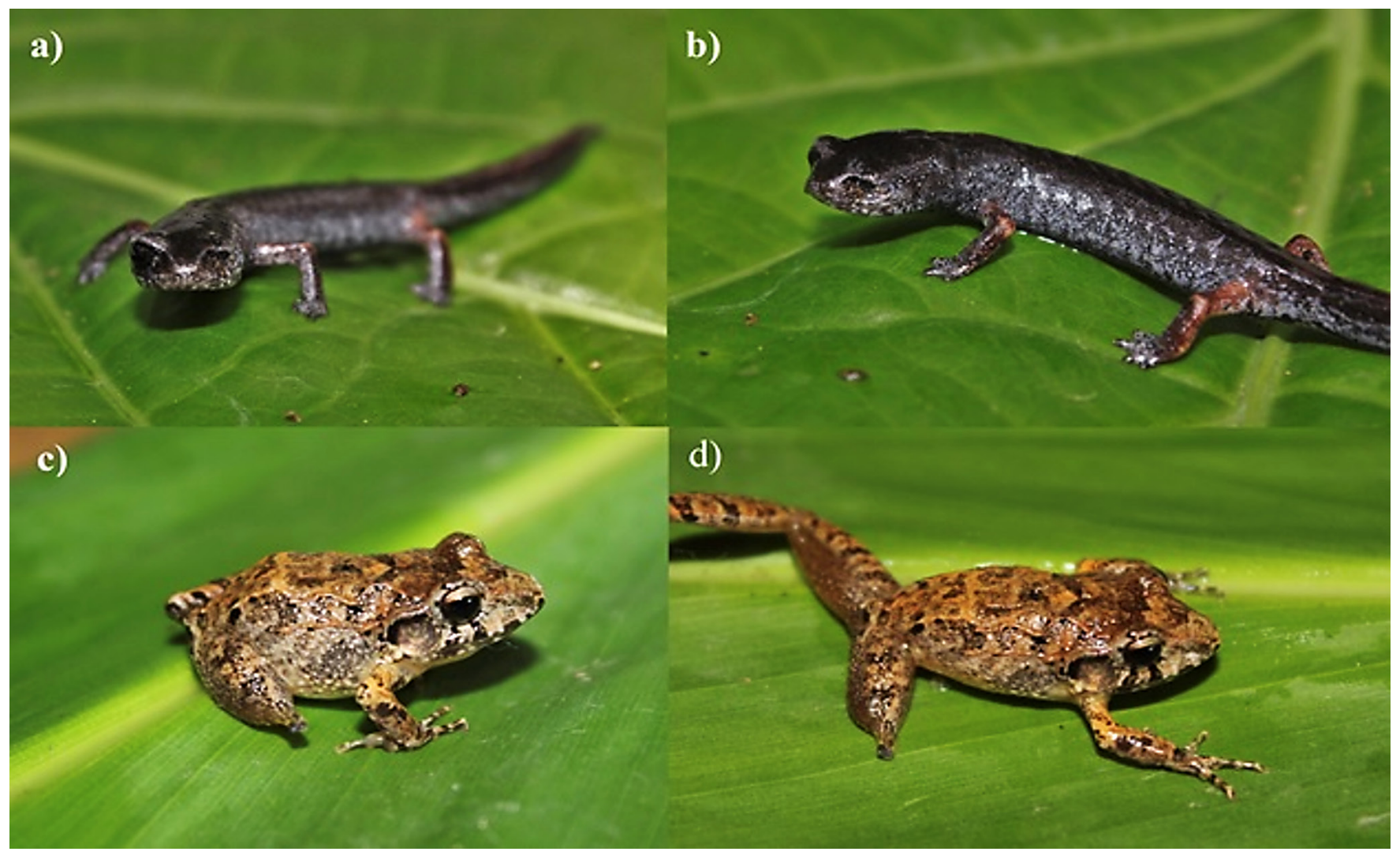

Figure 1. Front (a) and lateral (b) view of the case of microphthalmia in the left eye (globe and ocular cavity have reduced size) in an adult female Aquiloeurycea cafetalera (UTADC-9263). Lateral views ( $c$ and d) of the case of ectromelia of the right femur (absense of the lower portion of the leg) in an adult Craugator rhodopis (UTADC-9264). Photos: Juan Manuel Díaz-García.

Figura 1. Vista frontal (a) y vista lateral (b) del caso de microftalmia del ojo izquierdo (globo y cavidad ocular de tamaño reducido) en una hembra adulta de Aquiloeurycea cafetalera (UTADC-9263). Vistas laterales (c y d) del caso de ectromelia de fémur derecho (ausencia de la parte inferior de la pierna) en un individuo adulto de Craugastor rhodopis (UTADC-9264). Fotos: Juan Manuel Díaz-García.

WGS84, $1427 \mathrm{~m})$. El 13 de agosto de 2018 alrededor de las 21:00 h, capturamos una hembra adulta de A. cafetalera (longitud total $=54.4 \mathrm{~mm}, 1.4 \mathrm{~g}$ ) encontrada debajo de la hojarasca, la cual presentaba el globo y la cavidad ocular izquierda de un tamaño reducido (microftalmia; Figs. 1a y 1b). Posteriormente, el 14 de agosto de 2018 alrededor de las 20:00 h, capturamos un individuo adulto de $C$. rhodopis (longitud hocico-cloaca $=24.1$ $\mathrm{mm}, 1.3 \mathrm{~g}$ ) posado sobre la hojarasca, el cual no presentaba la sección inferior de la pierna derecha (ectromelia de fémur; Figs. 1c y id). Identificamos y verificamos las anormalidades siguiendo la Guía de campo para malformaciones de ranas y sapos con interpretación radiográfica (Meteyer, 2000). Meteyer (2000) indica que en la ectromelia de fémur, el fémur está presente pero termina en la diáfisis femoral (cuerpo del hueso) y no hay evidencia de articulación (rodilla). Mientras que la microftalmia se refiere a la presencia de un ojo de menor tamaño que el otro. Medimos, pesamos y fotografiamos los individuos, posteriormente los liberamos en el sitio de captura. Debido a que no contábamos con permiso de colecta, depositamos las fotografías en la Colección Digital de Vertebrados de la Universidad de Texas en Arlington (UTADC).

Las anormalidades que reportamos en A. cafetalera y $C$. rhodopis fueron observadas durante un estudio a largo plazo en el que monitoreamos las poblaciones de anfibios en pastizales ganaderos y bosques secundarios, además del remanente de bosque de niebla. Después de un esfuerzo de muestreo igual a 540 horas-persona, encontramos un total de 37 individuos de $A$. cafetalera y 323 individuos de C. rhodopis, pero solo uno de cada especie presentó una anormalidad. Esta baja incidencia difiere con otros estudios donde se han reportado incidencias que varían entre el 9 y $50 \%$ de los anfibios de una población (Johnson et al., 2006; Aguillón-Gutiérrez \& Ramírez-Bautista, 2015; SotoRojas et al., 2017). Sin embargo, resalta que observamos ambos 
casos de anormalidades en el interior de un bosque de niebla conservado, a diferencia de otros estudios en los que se describen anormalidades principalmente en ambientes modificados, como los cultivos agrícolas y las zonas urbanas (Oullet et al., 1997; Peltzer et al., 2011; Aguillón-Gutiérrez \& Ramírez-Bautista, 2015; Soto-Rojas et al., 2017).

La presencia de anormalidades puede afectar la supervivencia y la adecuación de los anfibios, debido a que incrementa la probabilidad de depredación, y reduce la eficiencia de forrajeo y la adquisición de pareja (Johnson et al., 2006). Por lo tanto, el incremento en el número de casos de anormalidades morfológicas a nivel mundial puede considerarse como una amenaza para los anfibios (Blaustein \& Johnson, 2003). Las observaciones que reportamos en esta nota sientan las bases para realizar más estudios en la región, en los que se puedan determinar los factores que ocasionan anormalidades morfológicas, así como definir con mayor certeza la tasa de incidencia y los efectos que pudieran tener sobre las poblaciones de anfibios que habitan el interior del bosque de niebla.

Agradecimientos.- A Ricardo Romero González y los socios de Las Cañadas por otorgar el permiso para realizar el trabajo de campo en sus terrenos, y por todas las facilidades que proporcionaron durante la estancia. A Antonio Reyes López por ayudarnos como guía en campo. A Eduardo Pineda por sus comentarios durante la elaboración de la nota. A Carl J. Franklin por catalogar las fotografías. Este trabajo fue financiado por el proyecto de Ciencia Básica del Consejo Nacional de Ciencia y Tecnología (CONACYT) "Atributos funcionales de especies arbóreas y los escenarios para la restauración del paisaje de bosque mesófilo de montaña en el Centro de Veracruz" No. CB2014-238831-B.

\section{LITERATURA CITADA}

Aguillón-Gutiérrez, D.R. \& A. Ramírez-Bautista. 2015. Anomalías frecuentes en una población de Hyla plicata (Anura: Hylidae) expuesta a plomo y fierro durante el desarrollo postembrionario. Biología Ciencia y Tecnología 8:515-529.

Ankley, G.T., S.J. Degitz, S.A. Diamond \& J.E. Tietge. 2004. Assessment of environmental stressors potentially responsable for malformations in North American anuran amphibians. Ecotoxicology Environmental Safety 58:7-17.

Barragán-Ramírez, J.L. \& J.L. Navarrete-Heredia. 2011. Primer registro de un caso de malformaciones en Lithobates neovolcanicus (Hillis y Frost, 1985) (Anura: Ranidae). Acta Zoológica Mexicana 27:837-841.
Blaustein, A.R. \& P.T. Johnson. 2003. The complexity of deformed amphibians. Frontiers in Ecology and the Environment 1:87-94.

Cruz-Pérez, M.S., J.A. Rangel-Hernández, O. Roldan-Padron, G.A. Soto-Alonso, U. Padilla-García \& U.O. García-Vázquez. 2009. Presencia de malformaciones en Ambystoma velasci en Alameda del Rincón, Querétaro, México. Boletín de la Sociedad Herpetológica Mexicana 17:92-96.

Gray, B.S. \& M. Lethaby. 2010. Observations of limb abnormalities in amphibians from Erie County, Pennsylvania. Journal of Kansas Herpetology 35:14-16.

Domínguez-Moreno, L.A., V. Vásquez-Cruz, A. Reynoso-Martínez, \& N.M. Cerón-de la Luz. 2018. Dos casos de anomalías macroscópicas en Rhinella horribilis (Anura: Bufonidae) en Veracruz, México. Boletín de la Asociación Herpetológica Española 29:153-154.

Johnson, P.T. \& K.B. Lunde. 2005. Parasite infection and limb malformations: a growing problem in amphibian conservation. Pp. 124-138. En M. Lannoo (Ed). Amphibian declines: the conservation status of United States species. University of California Press, Berkeley, USA.

Johnson, P.T., K.B. Lunde, R.W. Haight, J. Bowerman \& A.R. Blaustein. 2001. Ribeiroia ondatrae (Trematoda: Digenea) infection induces severe limb malformations in western toads (Bufo boreas). Canadian Journal of Zoology 79:370-379.

Johnson, P.T., E.R. Preu, D.R. Sutherland, J.M. Romansic, B. Han, \& A.R. Blaustein. 2006. Adding infection to injury: synergistic effects of predation and parasitism on amphibian malformations. Ecology 87:2227-2235.

Meteyer, C.U. 2000. Field guide to malformations of frogs and toads with radiographic interpretations. Biological Science Report, USA.

Monroy-Vilchis, O., L.L. Parra-López, T. Beltrán-León, J.A. Lugo, A. Balderas \& M.M Zarco-González. 2015. Morphological abnormalities in anurans from central Mexico: A case study (Anura: Ranidae, Hylidae). Herpetozoa 27:115-121.

Oullet, M., J. Bonin, J. Rodrigue, J.L. DesGranges \& S. Lair. 1997 Hindlimb deformities (ectromelia, ectrodactyly) in free-living anurans from agricultural habitats. Journal of Wildlife Diseases 33:95-104 
Peltzer, P.M., R.C. Lajmanovich, L.C. Sánchez, A.M. Attademo, C.M. Junges, C.L. Bionda, A.L. Martino \& A. Basso. 2011. Morphological abnormalities in amphibian populations from the mid-eastern region of Argentina. Herpetological Conservation and Biology 6:432-442.

Robles-Mendoza, C., C. García-Basilio, S. Cram-Heydrich, M. Hernández-Quiroz \& C. Vanegas-Pérez. 2009. Organophosphorus pesticides effect on early stages of the axolotl Ambystoma mexicanum (Amphibia: Caudata). Chemosphere 74:703-710.
Soto-Rojas C., I. Suazo-Ortuño, J.A. Montoya-Laos \& J. Alvaro-Díaz. 2017. Habitat quality affects the incidence of morphological abnormalities in the endangered salamander Ambystoma ordinarium. PLOS ONE 12(8):1-15. 